\section{Consumo de doces, refrigerantes e bebidas com adição de açúcar entre adolescentes da rede pública de ensino de Piracicaba, São Paulo}

\section{Consumption of sweets, soft drinks and sugar-added beverages among adolescents from public schools in Piracicaba, Sao Paulo}

\section{Marina Bueno do Carmo' \\ Natacha Toral ${ }^{2}$ \\ Marina Vieira da Silva ${ }^{3}$ \\ Betzabeth Slater ${ }^{4}$}

'Graduanda do curso de Nutrição da Faculdade de Saúde Pública da Universidade de São Paulo, bolsista de iniciação científica pelo Conselho Nacional de Desenvolvimento Científico e Tecnológico (CNPq), processo 111929/2004-0

${ }^{2}$ Nutricionista, especialista pela Universidade Federal de São Paulo, mestranda da Faculdade de Saúde Pública da Universidade de São Paulo;

${ }^{3}$ Professora doutora do Departamento de Agroindústria, Alimentos e Nutrição da Escola Superior de Agricultura Luiz de Queiroz / Universidade de São Paulo (ESALQ/USP);

${ }^{4}$ Professora doutora do Departamento de Nutrição da Faculdade de Saúde Pública da Universidade de São Paulo

Financiamento: Este estudo está vinculado ao projeto intitulado "Consumo dietético e atividade física como determinantes das mudanças do Índice de Massa Corporal de uma coorte de adolescentes matriculados na rede pública de ensino da cidade de Piracicaba, São Paulo", financiado pela Fundação de Amparo à Pesquisa do Estado de São Paulo (FAPESP) sob o protocolo 02/9521-9.

Correspondência: Marina Bueno do Carmo. Departamento de Nutrição da Faculdade de Saúde Pública da Universidade de São Paulo. Av. Dr. Arnaldo 715. Cerqueira César. CEP: 01246-904. São Paulo/SP. Telefone/FAX: (11) 3066-7705/ 3066-7701. Ramal:243.E-mail: marinabc@usp.br

\section{Resumo}

Objetivo: Descrever as práticas alimentares de adolescentes quanto à ingestão energética, distribuição de macronutrientes na dieta e porções consumidas de doces, refrigerantes e bebidas com adição de açúcar. Metodologia: Foi avaliada uma amostra de adolescentes matriculados em escolas da rede pública de ensino de Piracicaba, São Paulo. O consumo alimentar foi avaliado por um Questionário de Freqüência Alimentar. Para a comparação do consumo com a recomendação de ingestão de energia e de macronutrientes, utilizaram-se as Dietary Reference Intakes. A análise do consumo de doces baseou-se nas recomendações do guia alimentar norte-americano. Dados de consumo de refrigerantes e bebidas com adição de açúcar foram avaliados em comparação com outros estudos. Resultados: O estudo incluiu 390 adolescentes. Apenas 6,2\% destes apresentaram consumo energético em conformidade com o intervalo preconizado e $83,8 \%$ revelaram ingestão energética acima dos valores propostos. Expressiva parcela apresentou consumo de carboidratos e proteínas de acordo com o recomendado, mas foi verificado consumo alto de lipídios em $36,7 \%$ da amostra. Comprovou-se um consumo médio de 3,8 porções diárias de doces, sendo que $78,2 \%$ dos entrevistados ultrapassaram a recomendação máxima. O consumo médio diário alcançou aproximadamente $230 \mathrm{ml}$ e $550 \mathrm{ml}$ de refrigerante e bebidas com adição de açúcar, respectivamente. Conclusão: Devido à alarmante inadequação das práticas alimentares observadas entre os adolescentes, devem ser adotadas estratégias educativas que enfatizem a redução do consumo de açúcares e os benefícios da adoção de uma dieta equilibrada.

Palavras-chave: Adolescentes. Bebidas com adição de açúcar. Doces. Refrigerantes. Consumo alimentar. 
Abstract

Objective: To describe the dietary patterns of adolescents in relation to energy intake, distribution of macronutrients in diet and consumption of portions of sweets, soft drinks and sugar-added beverages. Methodology: A sample of adolescents enrolled in public schools in Piracicaba, São Paulo, was evaluated. A Food Frequency Questionnaire evaluated dietary intake. The $\mathrm{Di}$ etary Reference Intakes was used to compare energy and macronutrient intake. The analysis of sweets intake was based on the recommendations of North-American guidelines. Information about intake of soft drinks and sugar-added beverages was evaluated and compared to previous studies. Results: The study included 390 adolescents. Only $6.2 \%$ of them complied with the recommended energy intake and $83.8 \%$ presented consumption above the estimated level. Most of the adolescents presented carbohydrate and protein intake between recommended levels, but $36.7 \%$ showed a high fat intake. Average intake of sweets was 3.8 portions a day and $78.2 \%$ of the adolescents interviewed exceeded the maximum recommended. The daily average intake reached approximately $230 \mathrm{~mL}$ of soft drinks and $550 \mathrm{~mL}$ of sugar-added beverages. Conclusion: Due to the alarmingly inadequate dietary practices observed among adolescents, educational measures must be taken in order to emphasize the reduction in sugar intake and the benefits gained from following a balanced diet.

Keywords: Adolescents. Sugar added beverages. Sweets. Soft drinks. Dietary intake.

\section{Introdução}

A adolescência é um período da vida caracterizado por intensas mudanças biológicas, psicológicas e sociais que podem interferir no consumo alimentar deste grupo populacional ${ }^{1}$. A dieta de adolescentes caracteriza-se pela preferência de alimentos com elevado teor de gordura saturada, colesterol e substancial quantidade de sódio e carboidratos refinados, representados muitas vezes pela ingestão de batatas fritas, alimentos de origem animal fritos e bebidas com adição de açúcar ${ }^{2-4}$.

Estudos sobre a alimentação de grupos de adolescentes brasileiros indicam a ocorrência de inadequação alimentar com excesso de açúcares e gorduras ${ }^{5-7}$. Um aspecto relevante da dieta dos adolescentes é o consumo excessivo de doces e bebidas com adição de açúcar (incluindo refrigerantes). Segundo Fisberg et al. ${ }^{8}$, este é um problema comum no mundo todo.

Ao analisar a alimentação de adolescentes, Garcia et al. ${ }^{9}$ constataram um consumo elevado de bebidas gaseificadas e alimentos ricos em açúcar. Apesar da disponibilidade de alimentos saudáveis como lanche escolar, os adolescentes têm preferência por bolachas, batatas fritas, pizzas, refrigerantes e chocolates ${ }^{8}$.

Dados do Departamento de Agricultura dos Estados Unidos (USDA) mostraram um aumento de $500 \%$ no consumo de refrigerantes nos últimos 50 anos. Os refrigerantes constituem a maior fonte de açúcar adicionado na dieta. Atualmente, metade dos americanos e a maioria dos adolescentes (65\% das meninas e $74 \%$ dos meninos) consomem refrigerantes diariamente ${ }^{10}$.

Pesquisa implementada nos Estados Unidos, conduzida por Nielsen e Popkin ${ }^{11}$, analisou a tendência do consumo de bebidas (leite, suco de frutas, refrigerantes, bebidas à base de frutas, café, chá e bebidas alcoólicas) tendo por base o período entre 1977 e 2001. Os autores verificaram que houve um aumento na obtenção de energia proveniente de refrigerantes e bebidas à base de frutas. 
A tendência apresentada no consumo de alimentos ricos em açúcares simples é motivo de preocupação tendo em vista os efeitos deletérios destes alimentos quando consumidos de forma desarmoniosa em relação ao conjunto da alimentação ${ }^{6}$. A ingestão energética excessiva pode ser proveniente do aumento da oferta de alimentos em grandes quantidades, podendo repercutir sobre o aumento da prevalência de excesso de peso ${ }^{12,13}$.

Da Veiga et al. ${ }^{14}$ observaram um aumento alarmante das taxas de excesso de peso em adolescentes brasileiros. Os autores compararam os dados do Estudo Nacional de Despesa Familiar (ENDEF), realizado em 1974-1975, e da Pesquisa sobre Padrões de Vida (PPV), de 1996-1997 e constataram que o excesso de peso praticamente triplicou entre os meninos (de $2,6 \%$ para $11,8 \%$ ) e meninas nesse período (de 5,8\% para $15,3 \%$ ). Acredita-se que o aumento no número de porções consumidas possa ter contribuído para o aumento das taxas de obesidade também entre adolescentes, situação que é considerada como um dos principais problemas de saúde pública da atualidade em diversos países, inclusive no Brasil.

Assim, ressalta-se a importância de estudos sobre as práticas alimentares características desse estágio da vida, enfatizando o número de porções usualmente consumidas por esta população, a fim de se obter parâmetros da realidade atual. O objetivo deste estudo foi descrever as práticas alimentares de alunos adolescentes matriculados em escolas da rede pública da cidade de Piracicaba, São Paulo, em relação à ingestão energética, à distribuição de macronutrientes na dieta e ao número de porções consumidas de doces, refrigerantes e bebidas com adição de açúcar.

\section{Metodologia}

Trata-se de um estudo observacional de corte transversal, no qual foram incluídos adolescentes com idade mínima de 10 anos, de ambos os gêneros, matriculados em escolas da rede pública de ensino da cidade de Piracicaba. As entrevistas, realizadas durante o mês de novembro de 2004, foram conduzidas em espaço reservado em cada escola, durante a jornada de aula. Os participantes foram submetidos a entrevistas pessoais, conduzidas por pesquisadores treinados, com duração aproximada de 60 minutos. O presente estudo foi aprovado pelo Comitê de Ética da Faculdade de Saúde Pública.

Para o cálculo da amostra, foram consideradas as seguintes informações: prevalência de sobrepeso em população semelhante (15\%); erro tipo I de $5 \%$ e de erro tipo II de $20 \%{ }^{15}$.

O procedimento de amostragem foi do tipo aleatório simples, estratificado por séries ( $1^{\mathrm{a}}$ a $4^{\mathrm{a}}$ série e $5^{\mathrm{a}}$ a $8^{\mathrm{a}}$ série), sendo realizado em duas etapas. Na primeira etapa, as unidades primárias de amostragem (UPA), que correspondem às escolas do município, foram ordenadas segundo as regiões. Foram sorteadas 11 escolas, considerando que dez estavam localizadas em zonas urbanas e uma em zona rural.

Na segunda etapa da amostragem, foram ordenadas as unidades secundárias de amostragem, que correspondem às classes (séries) das escolas. Em cada UPA, as classes foram novamente sorteadas por amostragem aleatória simples. Em cada classe selecionada, realizou-se um sorteio de determinado número de alunos, dependendo do porte da escola.

Considerando que seria esperado identificar os alunos com idade mínima de 10 anos a partir da $4^{\text {a }}$ série, deveriam ser sorteados 130 alunos de $4^{\text {a }}$ série, 86 de $5^{\text {a }}$ série, 78 de $6^{a}$ série, 84 de $7^{a}$ série e 42 de $8^{a}$ série, totalizando uma amostra estimada de 420 alunos.

Foi realizado um estudo-piloto que possibilitou adaptações no formato do questionário, de modo a favorecer a compreensão das perguntas e maior agilidade na realização da entrevista. A escola na qual foi realizado o estudo-piloto não foi incluída na amostra do estudo principal. A seguir, são descritas as variáveis investigadas: 
- Variáveis demográficas: coletaram-se informações relativas ao gênero e à idade do participante.

- Consumo alimentar: a avaliação do consumo habitual foi realizada por meio da aplicação do Questionário de Freqüência Alimentar para Adolescentes (QFAA), validado por Slater et $a^{2}$. Tratase de um questionário de freqüência alimentar semi-quantitativo, contendo 94 alimentos e sete opções de consumo: nunca; menos de uma vez ao mês; de 1 a 3 vezes no mês; 1 vez por semana; de 2 a 4 vezes por semana; 1 vez ao dia; 2 ou mais vezes ao dia. Para facilitar a obtenção de respostas dos entrevistados foi utilizado um material fotográfico elaborado especialmente para a pesquisa, apresentando diferentes utensílios, comumente adotados na alimentação, como pratos, copos e colheres.

Foram analisados os dados referentes à ingestão de energia, proteína, carboidratos e lipídios totais. Para a análise da adequação energética das dietas e do consumo dos macronutrientes, foram utilizadas as Dietary Reference Intakes (DRIs) ${ }^{16}$. A avaliação do consumo energético foi elaborada comparando-se a ingestão energética obti- da para cada adolescente com a necessidade energética estimada. Para o cálculo desta, foram adotadas as fórmulas para crianças e adolescentes de 9 a 18 anos $^{16}$ : EER= 88,5 - 61,9 x idade (anos) + 1,13 x [peso (kg) $\mathrm{x} 26,7+903 \mathrm{x}$ altura $(\mathrm{m})]+25$, para os meninos e EER $=135,3-30,8 x$ idade (anos) $+1,16$ $\mathrm{x}$ [peso $(\mathrm{kg})$ x $10+934$ x altura $(\mathrm{m})]+25$, para as meninas, sendo que EER= Estimated Energy Requirement (Requerimento Energético Estimado). Os desvios-padrão utilizados foram 58kcal para os meninos e $68 \mathrm{kcal}$ para as meninas. Foram considerados como faixa de adequação os valores compreendidos entre dois desvios-padrão acima e abaixo do estimado ${ }^{16}$. Com relação à ingestão dos macronutrientes, as recomendações utilizadas para o cálculo da adequação foram: 45 a $65 \%$ do valor calórico total (VCT) provenientes de carboidratos, 10 a $30 \%$ do VCT de proteínas e 25 a $35 \%$ do VCT de lipídios. Esses dados correspondem à faixa etária de 4 a 18 anos $^{16}$. Foi investigado o número médio de porções diárias consumidas em relação aos seguintes grupos alimentares: doces, refrigerantes e bebidas com adição de açúcar. Os alimentos e/ou preparações incluídos em cada um desses grupos estão registrados na Tabela 1.

Tabela 1 - Descrição dos alimentos e preparações que integram os grupos alimentares estudados. Table 1 - Description of food and preparations included in the dietary groups studied.

\begin{tabular}{lll}
\hline GRUPO ALIMENTAR & ALIMENTOS INTEGRANTES & QUANTIDADE \\
\hline Açúcares e doces & Achocolatado em pó & 2 colheres de sopa rasas \\
& Açúcar de adição & 2 colheres de sobremesa \\
& Balas & 2 unidades \\
& Biscoitos recheados & 7 unidades \\
& Chocolate/brigadeiro & 1 tablete pequeno/ 3 unidades pequenas \\
& Croissant de chocolate & 1 unidade média \\
& Doces de frutas (goiabada, marmelada, doce & 1 fatia fina/ 1 unidade média \\
& de abóbora, etc) & \\
& Sobremesas tipo mousse & 1 taça/ 1 pote \\
& Sorvete de massa ou palito & 2 bolas/ 1 unidade \\
\hline Refrigerantes & Refrigerante normal & 1 1/2 copo de requeijão/ 1 lata \\
\hline Bebidas com adição & Chá mate com sabor & 1 lata \\
de açúcares & Suco de frutas (abacaxi, laranja, mexerica, mamão, & 1 copo de requeijão \\
& melão, melancia) com açúcar & 1 copo de requeijão \\
& Limonada e laranjada & 1 copo de requeijão \\
& Sucos naturais com leite e vitaminas de frutas & 1 copo de requeijão \\
\hline
\end{tabular}


As porções foram determinadas a partir de uma lista de alimentos, proveniente de dados secundários. Foi definido o tamanho das porções segundo a metodologia proposta por Block et al. ${ }^{17}$.

A análise do número de porções consumidas pelos adolescentes baseou-se nas recomendações do Dietary Guidelines for Americans ${ }^{18}$ para doces, que sugerem o consumo máximo de duas porções ao dia. Para avaliar o consumo em relação a refrigerantes e bebidas com adição de açúcar, os dados observados foram descritos em diversos estudos ${ }^{24,29,30}$.

Para a análise da composição nutricional foi adotado o software Dietsys versão $4.01^{19}$. Foram excluídos da análise os indivíduos cuja ingestão energética apresentava-se inferior a 500 calorias (ou 5 itens do QFAA) ou superior a 7.000 calorias (ou 51 itens do QFAA $)^{10}$. A utilização do software Epidata, versão $3.02^{20}$, permitiu a construção dos demais dados.

Após a realização do teste de Kolmogorov-Smirnov, optou-se pela adoção de testes não-paramétricos com vistas às análises envolvidas neste estudo. Foram calculadas medidas de tendência central e de dispersão, além de comparar o consumo dos adolescentes, de acordo com o gênero por meio do teste de MannWhitney. Adotou-se o grau de significância estatística de 5\%. A análise foi realizada com o auxílio do programa Statistical Package for the Social Sciences versão $10.0^{21}$. Os resultados serão apresentados como média (intervalo de confiança de $95 \%)$.

\section{Resultados}

Foram entrevistados 431 adolescentes e excluídos 41 participantes, distribuídos nas seguintes situações: consumo diário de menos de 5 itens do QFAA ( $\mathrm{n}=2)$; consumo diário de mais de 51 itens do QFAA $(n=7)$; consumo superior a 7000kcal $(n=23)$ e dados incompletos em relação a alguma variável $(n=9)$. A amostra final incluiu, portanto, 390 participantes com idade entre 10 e 17 anos. A média de idade foi de 12,4 anos, sendo significativamente maior entre os meninos (12,6 vs. 12,2 anos; $\mathrm{p}=0,017$ ). A distribuição dos adolescentes segundo gênero revelou um discreto predomínio do gênero feminino $(53,6 \%)$.

Apenas 6,2\% dos adolescentes apresentaram ingestão energética em conformidade com o intervalo preconizado. Observou-se que $83,8 \%$ da amostra apresentaram ingestão energética acima dos valores propostos (Figura 1). A participação dos macronutrientes no valor calórico total apresentou-se de acordo com as recomendações para a maioria dos adolescentes. Destaca-se, no entanto, o consumo eleva-

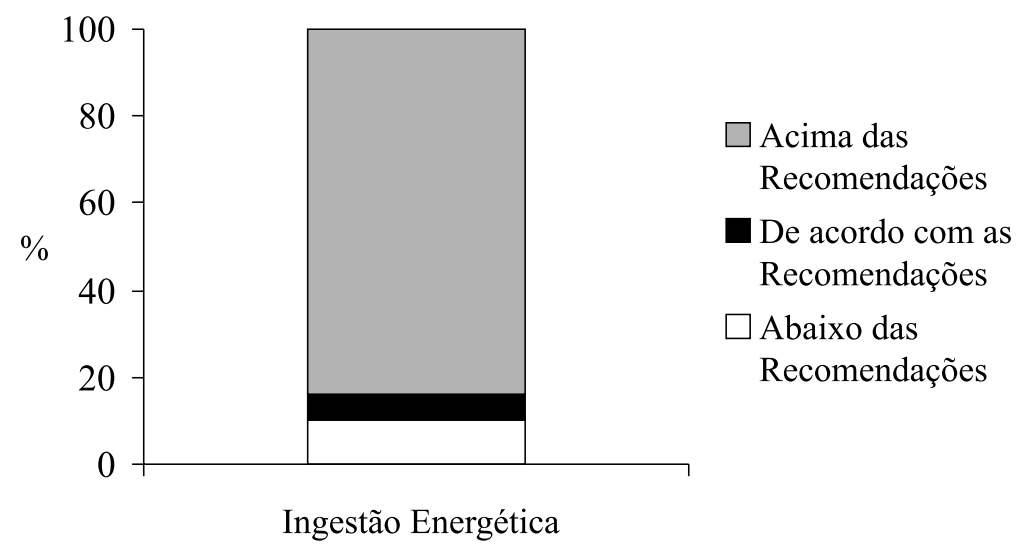

Figura 1- Adequação das dietas dos adolescentes, de acordo com a ingestão energética. Piracicaba, São Paulo, 2004.

Figure 1- Diet adequacy for adolescents, according to energy intake. Piracicaba, Sao Paulo, 2004. 
do de lipídios, observado entre 36,7\% dos adolescentes (Figura 2). Não foi encontrada diferença significativa entre os dados obtidos para os distintos gêneros, quanto à ingestão energética e quanto à participação relativa de macronutrientes na dieta $(\mathrm{p}>0,05)$.

Foi constatado um consumo elevado de doces entre os entrevistados: em média, 3,8 porções diárias (Tabela 2). Verificou-se que $78,2 \%$ dos adolescentes ultrapassaram a recomendação de ingestão máxima de 2 porções/dia. Consumo médio diário de aproximadamente $230 \mathrm{ml}$ e $550 \mathrm{ml}$ de refrigerante e bebidas com adição de açúcar, respectivamente, foi identificado entre os adolescentes. Não foram observadas diferenças significativas de consumo dos três grupos alimentares in- vestigados, tendo por referência o gênero ( $>>0,05)$.

\section{Discussão}

Nas últimas décadas, e em vários países, tem sido observada uma rápida mudança na composição dietética, que está associada a fatores econômicos, demográficos, sociais, epidemiológicos e nutricionais ${ }^{22}$. Segundo a recente Pesquisa de Orçamentos Familiares (POF) realizada pelo Instituto Brasileiro de Geografia e Estatística (IBGE) entre 2002 e 2003, foi identificado um desequilíbrio entre os alimentos disponíveis nos domicílios, devido à presença excessiva de açúcar, com importante incremento no consumo de refrigerantes ${ }^{23}$. O presente estudo identificou um quadro se-

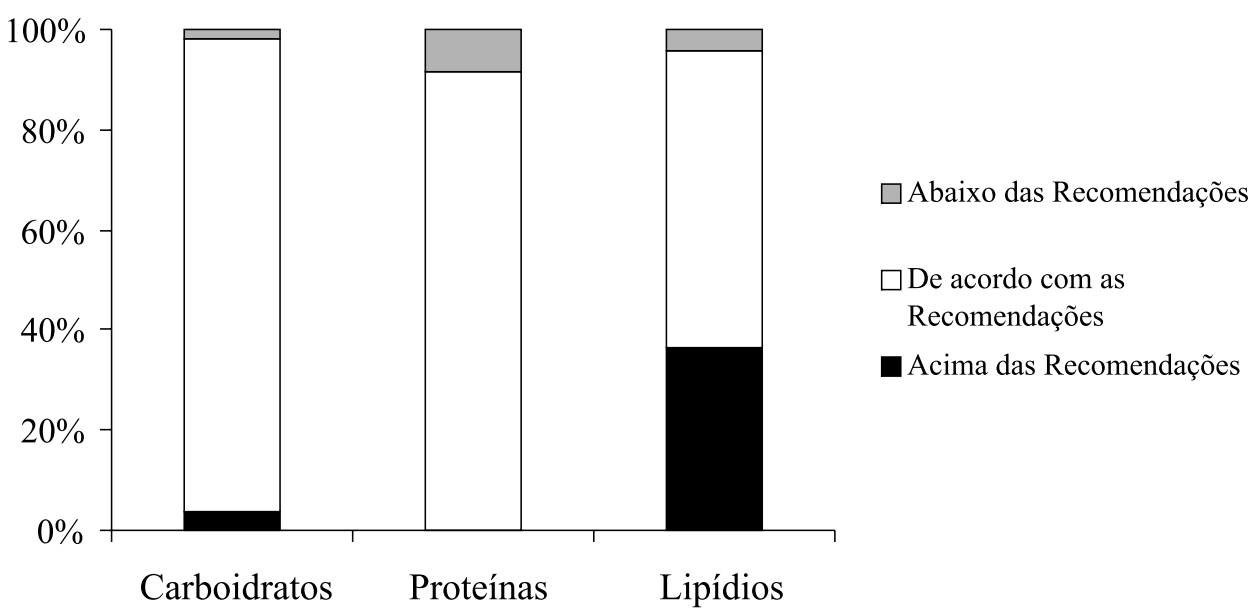

Figura 2 - Adequação das dietas dos adolescentes, de acordo com o consumo de macronutrientes. Piracicaba, São Paulo, 2004.

Figure 2 - Diet adequacy for adolescents, according to macronutrient intake. Piracicaba, Sao Paulo, 2004.

Tabela 2 - Consumo observado entre os adolescentes em relação aos grupos alimentares selecionados. Piracicaba, São Paulo, 2004.

Table 2 - Adolescents' intake regarding selected dietary groups. Piracicaba, Sao Paulo, 2004.

\begin{tabular}{lcccccc}
\hline GRUPO ALIMENTAR & \multicolumn{2}{c}{ Total $(n=390)$} & \multicolumn{2}{c}{ Meninos $(n=181)$} & \multicolumn{2}{c}{ Meninas $(n=209)$} \\
& média & IC & média & IC & média & IC* \\
\hline Açúcares e doces (porções/dia) & 3,7 & $3,5-3,9$ & 3,8 & $3,5-4,1$ & 3,7 & $3,5-4,0$ \\
Refrigerantes (mL/dia) & 226,3 & $206,1-246,5$ & 233,7 & $203,7-263,7$ & 219,8 & $192,3-247,4$ \\
Bebidas açucaradas (mL/dia) & 554,2 & $517,7-590,6$ & 567,0 & $512,6-621,3$ & 543,1 & $493,5-592,6$ \\
\hline
\end{tabular}

* IC: Intervalo de confiança (95\%); Cl:Confidence Interval 
melhante entre adolescentes brasileiros, em relação ao consumo de doces, refrigerantes e bebidas com adição de açúcar.

No entanto, comparações com outros estudos de avaliação do consumo alimentar de adolescentes são limitadas. As metodologias adotadas freqüentemente não são compatíveis e são escassos os trabalhos que descrevem as práticas alimentares desse estágio de vida. Com relação à ingestão energética, os dados obtidos diferem dos encontrados por Kazapi et al. ${ }^{24}$. Esses autores avaliaram adolescentes de escolas públicas e privadas do município de Florianópolis, Santa Catarina ${ }^{24}$ e observaram que aproximadamente $50 \%$ da amostra apresentaram ingestão energética insuficiente. De maneira oposta, no presente estudo, este valor foi de apenas $10,7 \%$. A maior parte dos adolescentes apresentou ingestão de energia acima das recomendações.

Apesar da adoção de procedimento metodológico distinto, os resultados obtidos por Kazapi et al. ${ }^{24}$ em relação ao consumo de macronutrientes foram semelhantes aos identificados por meio do presente estudo. Em ambas as pesquisas, mais da metade dos adolescentes revelou um consumo adequado de carboidratos e elevada participação relativa de lipídios. No estudo de Kazapi et al. ${ }^{24}$ foi identificada proporção de adolescentes com consumo mais elevado, apenas no tocante à proteína, em comparação com os achados do presente estudo.

Assim como o observado neste estudo, outros autores ressaltam o elevado consumo de doces como uma prática comum entre adolescentes ${ }^{25-27}$. Acredita-se que nos Estados Unidos a ingestão de alimentos pobres em nutrientes represente atualmente mais de $30 \%$ da ingestão diária. Entre os principais participantes dessa proporção, são citados os refrigerantes, as balas, o açúcar adicionado e as sobremesas ${ }^{26}$.

O elevado consumo de doces e de bebidas com açúcar encontrado nesta pesquisa está em conformidade com o crescimento da participação de açúcar na dieta da população brasileira. Segundo os dados da POF, o teor de sacarose da dieta corresponde a $13,7 \%$ da energia total disponível, contra um máximo recomendado de $10 \%$ para a população adulta ${ }^{23}$.

O consumo médio diário de refrigerantes observado entre os entrevistados foi inferior ao encontrado por French et al. ${ }^{28}$. Os autores examinaram as tendências do consumo de refrigerantes pelos adolescentes norte-americanos com idade entre 6 e 17 anos e verificaram que a média de consumo aumentou de $148 \mathrm{~g}$ para $355 \mathrm{~g}$ no período de 1977/1978 e 1994/1998. No Brasil, também foi observado um fenômeno semelhante em relação ao consumo de tais bebidas, com um aumento que supera $490 \%{ }^{23}$. Observou-se que a participação relativa do refrigerante no total de energia da dieta aumentou de $0,43 \%$ segundo dados do Estudo Nacional de Despesa Familiar (ENDEF), realizado em 1974-1975, para $2,12 \%^{23}$.

O aumento da ingestão energética tem sido positivamente associado ao aumento do consumo de bebidas adoçadas ${ }^{29}$. Segundo Berkey et al..$^{30}$, é observado que o consumo excessivo de leite e sucos de frutas pode contribuir também para uma elevada ingestão energética. Mas tais bebidas apresentam uma composição nutricional mais adequada e, portanto, superior em comparação à observada em refrigerantes, tendo em vista que os últimos fornecem baixo conteúdo de micronutrientes e elevado teor calórico $^{30}$. Esse fato pode justificar a escassez de estudos que descrevam o consumo de bebidas com adição de açúcar.

Os achados descritos são preocupantes, considerando-se a relação entre a adoção de dietas desequilibradas e a manifestação de doenças crônicas. Sabe-se que a ingestão média de bebidas adicionadas de açúcar, incluindo os refrigerantes, pode contribuir para o ganho de peso, sendo associada ao desenvolvimento da obesidade na infância ${ }^{19,30}$.

$\mathrm{O}$ aumento na prevalência de obesidade tem sido observado nos Estados Unidos e em diversos países, entre todos os 
estágios de vida, com destaque para sua ocorrência entre pré-escolares e adolescentes. Tendo em vista que a obesidade na infância freqüentemente persiste na vida adulta, o aumento do sobrepeso e obesidade nas crianças é claramente o contribuinte mais expressivo da epidemia de obesidade identificada entre os adultos ${ }^{31}$. Não restam dúvidas quanto aos prejuízos na qualidade de vida futura dos indivíduos em tal situação e às perdas econômicas e sociais que o quadro envolve. A concomitância de fatores de risco durante o período de crescimento, como as dislipidemias e o aumento da resistência insulínica, está associada a um aumento da mortalidade no adulto devido ao possível desenvolvimento de doença arteriosclerótica, hipertensão e alterações metabólicas. Tal fato destaca a importância da identificação precoce do problema, devendo ser analisadas as variações ponderais desde a infância como prevenção e controle da permanência da obesidade em idades mais avançadas $^{32}$. Isto é, a prevenção e o tratamento da obesidade na infância são as melhores alternativas para evitar posteriores complicações de saúde ${ }^{27}$.

O presente estudo apresenta outras limitações. Sabe-se que a utilização de um Questionário de Freqüência Alimentar não permite inferências sobre adequação da dieta, já que compreende uma lista limitada de alimentos. Contudo, a referida análise teve como objetivo principal a elaboração de uma comparação com os valores propostos como ideais para uma alimentação saudável. Os dados apresentados também devem ser analisados com cautela, uma vez que a utilização do referido inquérito alimentar apresenta tendência à superestimativa do consumo alimentar. Por outro lado, é pouco provável que os resultados tenham sido alterados significativa- mente por esse fato, tendo em vista a semelhança encontrada com outros estudos implementados mais recentemente.

Considera-se ainda, que os Questionários de Freqüência Alimentar são delineados e conduzidos para uma determinada população. Por isso, os resultados obtidos neste estudo não podem ser extrapolados para a população brasileira.

Ressalta-se que as recomendações dietéticas estrangeiras foram consideradas como parâmetro de avaliação do consumo de doces devido à inexistência no Brasil até o momento de guias alimentares abrangentes que especifiquem o tema da alimentação saudável na adolescência.

\section{Conclusão}

Este estudo encontrou elevada ingestão energética e expressivo consumo de doces, bebidas com adição de açúcar e refrigerantes entre os adolescentes de Piracicaba. Esta alarmante inadequação está de acordo com os resultados encontrados entre adolescentes norte-americanos e a população adulta brasileira. Podese concluir que a dieta adotada nesse estágio de vida exige o desenvolvimento imediato de programas de intervenção nutricional. Devem ser adotadas estratégias educativas que enfatizem a redução do consumo de açúcares na alimentação e os benefícios decorrentes da adoção de uma dieta equilibrada. Tais medidas visam contribuir para a qualidade de vida dos adolescentes e a prevenção de agravos à saúde na vida adulta. Sugere-se que sejam realizadas outras pesquisas analisando a prática alimentar adotada na adolescência para a melhor avaliação dos presentes achados e para ampliar o conhecimento sobre as práticas alimentares inerentes a esse estágio da vida. 


\section{Referências}

1. Organización Mundial de la Salud. La Salud de los jóvenes: un reto y una esperanza. Ginebra: OMS; 1995.

2. Slater B, Fisberg RM, Philippi ST, Latorre MRDO. Validation of a semi-quantitative adolescents food frequency questionnaire applied at public school in São Paulo, Brazil. Eur J Clin Nutr 2003; 57: 629-35.

3. Andrade, R G., Pereira, R A. and Sichieri, R. Food intake in overweight and normal-weight adolescents in the city of Rio de Janeiro. Cad Saúde Pública 2003; 19 (5): 1485-95.

4. Garcia GCB, Gambardella AMD, Frutuoso MFP. Estado nutricional e consumo alimentar de adolescentes de um centro de juventude da cidade de São Paulo. Rev Nutr 2003; 16 (1): 41-50.

5. Gambardella AMG, Frutuoso MFP, Franchi C. Prática alimentar de adolescentes. Rev Nutr Campinas 1999; 12(1): 5-63.

6. Carvalho CMRG, Nogueira, AMT, Teles JBM, Paz, SMR, Sousa RML. Consumo alimentar de adolescentes matriculados em um colégio particular de Teresina, Piauí, Brasil. Rev Nutr Campinas 2001; 14(2): 85-93.

7. Albano RD, Souza SB. Ingestão de energia e nutrientes por adolescentes de uma escola pública. J Pediatr (Rio J) 2001; 77(6): 512-6.

8. Fisberg M, Bandeira CRS, Bonilha EA, Halpern G, Hirschbruch MD. Hábitos alimentares na adolescência. Pediatr Mod 2000; 36 (11): 724-34.

9. Garcia GCB, Gambardella AMD, Frutuoso, MFP. Estado nutricional e consumo alimentar de adolescentes de um centro de juventude da cidade de São Paulo. Rev Nutr Campinas 2003; 16(1): 41-50.

10. Ludwig DS, Peterson KE, Gortmaker SL. Relation between consumption of sugar-sweetened drinks and childhood obesity: a prospective, observational analysis. Lancet 2001; 357: 505-8.

11. Nielsen SJ, Popkin BM. Changes in Beverage Intake Between 1977 and 2001. Am J Prev Med 2004; 27 (3): 20510.

12. Nestlé M. Increasing portion sizes in American diets: More calories, more obesity. JAm Diet Assoc 2003; 103 (1): 39-40.

13. Smiciklas-Wright H, Michel DC, Mickle SJ, Goldman JD, Cook A. Foods commonly eaten in the United States, 1989-1991 and 1994-1996: Are portion sizes changing? $J$ Am Diet Assoc 2003; 103 (1): 41-7.

14. Da Veiga GV, Cunha AS, Sichieri R. Trends in overweight among adolescents living in the poorest and richest regions of Brazil. Am J Public Health 2004; 94 (9): 1544-8.

15. Popkin BM, Gordon-Larsen P. The nutrition transition: worldwide obesity dynamics and their determinants. Int $J$ Obes 2004; 28: S2-S9.
16. Institute of Medicine. Food and Nutrition Board. Dietary Reference Intakes for Energy, Carbohydrate, Fiber, Fat, Fatty Acids, Cholesterol, Protein, and Amino Acids (Macronutrients). Washington: National Academy Press, 2000. Disponível em <URL:http://www.nap.edu/> [acessado em 2005 fev 05]

17. Block G, Dresser CM, Hartman AM, Carroll MD. Nutrient sources in the American diet: quantitative data from the nhanes II survey. I. Vitamins and minerals. II. Macronutrients and fats. Am J Epidemiol 1985; 122: 13-40.

18. U. S. Department of Health and Human Services and U. S. Department of Agriculture. Dietary Guidelines for Americans, 2005. $6^{\text {th }}$ edition, Washington, DC: U. S. Government Printing Office, January 2005. Disponível em <URL:http://www.usda.gov/cnpp/DG2005/index.html> [acessado em 2005 jan 01]

19. HHHQ-DietSys Analysis Software, Version 4.01. National Cancer Institute; 1999.

20. Lauritsen JM, Bruus M, Myatt MA. An extended tool for validated data entry and documentation of data. Odense, Denmark: The EpiData Associationz; 2002.

21. Statistical Package for the Social Sciences for Windows Student Version/ SPSS. Release 10.0 Chicago: Marketing Department; 2000.

22. Popkin BM. The nutrition transition and obesity in the developing world. J Nutr 2001; 131(3): S 871-3.

23. [IBGE] Instituto Brasileiro de Geografia e Estatística. Pesquisa de Orçamentos Familiares (POF) 2002-2003: análise da disponibilidade domiciliar de alimentos e do estado nutricional no Brasil. Rio de Janeiro: IBGE, 2004. Disponível em <URL: http://www.ibge.gov.br/home/ estatistica/populacao/condicaodevida/pof/2002analise/ pof 2002analise.pdf> [acessado em 2005 mar 05].

24. Kazapi IL, Di Pietro PF, Avancini SRP, Freitas SFT, Tramonte VLCG. Consumo de energia e macronutrientes por adolescentes de escolas públicas e privadas. Rev Nutr 2001; 14: 27-33.

25. Nielsen SJ, Popkin BM. Patterns and Trends in Food Portion Sizes, 1977-1998. JAMA 2003; 289 (4): 450-3.

26. Muñoz KA, Krebs-Smith SM, Ballard-Barbash R, Cleveland LE. Food Intakes of US Children and Adolescents Compared With Recommendations. Pediatrics 1997; 100: 323- 9.

27. Nicklas TA, Elkasabany A, Srinivasan SR, Berenson G. Trends in nutrient intake of 10-year-old children over two decades (1973-1994): the Bogalusa Heart Study. Am J Epidemiol 2001; 153: 969-77.

28. French AS, Lin B, Guthrie JF. National trends in soft dink consumption among children and adolescents age 6 to 17 years: Prevalence, amounts, and sources, 1977/1978 to 1994/1998. JAm Diet Assoc 2003; 103 (10): 1326-31. 
29. Frary CD, Johnson RK, Wang MQ. Children and adolescents' choices of foods and beverages high in added sugars are associated with intakes of key nutrients and food groups. JAdolesc Health. 2004; 34 (1): 56-63.

30. Berkey CS, Rockett HRH, Field AE, Gillman MW, Colditz GA. Sugar-added beverages and adolescent weight change. Obes Res 2004; 12(5): 778-88.
31. Deckelbaum RJ, Williams CL. Childhood Obesity: The Health Issue. Obes Res 2001; 9(90004): S239-43.

32. Fisberg M. Atualização em Obesidade na Infância e Adolescência. São Paulo: Editora Atheneu; 2004.

Recebido em: 10/11/05

Versão reformulada reapresentada em: 06/03/06

Aprovado em: 21/03/06 